\title{
La estandarización en el currículo educativo: la punta del iceberg de la homogeneización
}

\section{The standardization in the curriculum educational: the tip of the iceberg of the homogenization}

Dra. Elsy Rodríguez-Revelo es Docente del Colegio Réplica Simón Bolívar de Guayaquil (Ecuador) (Rev_49@hotmail.com) (http://orcid.org/0000-0003-4486-0785 )

Recibido: 2017-01-30 / Revisado: 2017-04-16 / Aceptado: 2017-04-25 / Publicado: 2017-07-01

\section{Resumen}

Teniendo como referencia la literatura existente, con este artículo se pretende hacer una breve exposición de la estandarización curricular y su puesta en práctica en el campo educativo. Los estándares, tomados del campo empresarial, son considerados instrumentos necesarios para el aseguramiento de la calidad educativa. Sin embargo, las investigaciones empíricas y voces críticas en relación a su aplicabilidad en todos los contextos educativos, subrayan que los estándares homogenizan la cultura y unifican la enseñanza y que no son garantía de una mejora en los aprendizajes, ya que se tiende a dejar de lado la diversidad cultural existente en los centros educativos. Asimismo, los estándares influyen en la fragmentación y desigualdad académica: aptos (capaces) y los no aptos (poco capaces). Así pues, tanto las investigaciones relativas al tema, como los diferentes planteamientos de los autores, de manera implícita, y en algunos casos explícitamente, hacen un llamado a la reflexión para recuperar la enseñanza crítica pedagó- gica, en la cual se valoren las actitudes y aptitudes del individuo, como buenas prácticas para una sociedad del conocimiento de manera inclusiva.

Descriptores: Homogeneización, estándares educativos, cultura escolar, currículo, evaluación.

\begin{abstract}
Taking as a reference the existing literature, through this article intends to make a brief presentation of curriculum standardization and its implementation in the field of education. Standards, taken from the business field, are considered to be a necessary instrument for assurance of educational quality. However, empirical research, as well as critical voices in relation to their applicability, in all educational contexts, emphasize that these cultural homogenized and unify teaching, which does not guarantee an improvement in learning, since it tends to ignore cultural diversity in schools. Standards, taken from the business field, are considered to be a necessary instrument for assurance
\end{abstract}

Forma sugerida de citar: Rodríguez-Revelo, E. (2017). La estandarización en el currículo educativo: la punta del iceberg de la homogeneización. Alteridad, 12(2), 248-258. https://doi.org/10.17163/alt.v12n1.2017.10. 
of educational quality. However, empirical research, as well as critical voices in relation to their applicability, in all educational contexts, emphasize that these cultural homogenized and unify teaching, which does not guar-

\section{Introducción}

En este artículo se presentan algunos planteamientos acerca de la introducción de los estándares en el campo educativo. Estos estándares tienen el propósito de homogeneizar el currículo y su función de medidores de los aprendizajes en los estudiantes a través de las evaluaciones, pues "los números estandarizan y reubican lo local y lo personal dentro de sistemas abstractos de conocimientos" (Popkewitz, 2013, p. 53). Los temas tratados son objeto de un debate entre los profesionales y los teóricos de la educación. No obstante, ambas vertientes subrayan que la estandarización del currículo, así como la evaluación de los aprendizajes para medir el conocimiento adquirido por los alumnos buscan asegurar y controlar el qué y el para qué de la enseñanza, debido a su implicación ideológica, por parte de la macropolítica. Como contrapartida, el currículo estandarizado puede tener propósito homogeneizador de la cultura y la conducta del aprendiz a lo largo de la vida. En suma, este breve análisis plantea abordar que el resultado de un currículo estandarizado es la homogenización en los criterios de enseñanza y que esa homogenización puede tener posibles repercusiones a la hora de afrontar lo diverso en las aulas (Apple, 1986; Freire 1990; Giroux y McLaren, 1998; Popkewitz, 2013; Hargreaves y Fullan, 2014). Es decir, los estándares proyectan una visión clásica de la educación centrada en lo institucional. Por tanto, el conocimiento es la meta a alcanzar en todo el proceso de enseñanzaaprendizaje, sin contar con lo cotidiano y comunitario del individuo.

Existen grandes defensores de la diversidad cultural que se posicionan a favor de una pedagogía crítica y emancipadora, como Apple (1986), Freire (1990), Giroux y McLaren, (1998), antee an improvement in learning, since it tends to ignore cultural diversity in schools.

Keywords: Homogenization, standards education, culture school, curriculum, assessment.

Popkewitz (2013) y Hargreaves y Fullan (2014), entre otros. Esas voces cuestionan una educación que forma al individuo con base en una cultura única susceptible de vulnerar su identidad, pues ignoran la heterogeneidad de los educandos. Asimismo, las diferentes fuentes consultadas mantienen de manera implícita abierto el interrogante, pues no hay un consenso al respecto: ¿está respondiendo la educación a un interés público, sentido y aceptado por el conjunto de la sociedad, o forma parte de toda una maquinaria ideológica técnico-empresarial que busca desarrollo a costa de una pérdida de identidad cultural? Todas las opiniones y debates que se puedan abrir en torno al tema también dan sentido a la educación y proporcionan herramientas útiles para mejorar el quehacer educativo.

\section{La búsqueda de la homogenei- zación en el currículo: un para- lelogramo entre lo que debe enseñar el profesor y lo que debe aprender el estudiante}

Como guía para entender la primera parte de este análisis, partimos del hecho de ver en las transformaciones sociales, económicas, políticas y culturales, el pilar necesario sobre el cual se construyen los conocimientos, así como los procesos del saber. En otras palabras, el fin último de la educación es construir ventajas competitivas en los individuos, ya que el recurso básico para la sociedad es el conocimiento que debe ser aplicado en el campo laboral (Quijano, 2005), y su valor se mide con base en la productividad (ingeniería social).

Para hacer frente a esta realidad, tanto en el ámbito nacional como en el internacional se busca homogeneizar los procesos educativos (currículo, 
evaluaciones, entre otros), en general a favor del capital financiero. Por tanto, para alcanzar dicho cometido se hace uso de procesos técnicos-instrumentales (estándares) para homogenizar el currículo educativo y a su vez medir los aprendizajes alcanzados por los estudiantes, de tal manera que aseguren la calidad del producto humano que se insertará, en un futuro, en el mercado laboral (visión management de la educación). Es así que la educación queda vinculada al servicio de los grandes capitales para que los individuos participen a través de su desempeño laboral en materializar las políticas neoliberales desde las diferentes ramas del conocimiento: ciencias, ciencias de la salud, ciencias sociales y jurídicas, ingeniería y arquitectura, entre otras (Muñoz, Córdoba, Villareal, 2014). Este desempeño es catalogado por Casassus (1997) como competencia, la cual se alcanza con una formación o preparación de los estudiantes para intervenir de manera eficaz en un contexto laboral. Por tanto, esta competencia requiere ser demostrable a través de las evaluaciones, por ejemplo las pruebas PISA.

La estandarización curricular busca ordenar los contenidos que se deberían desarrollar en diferentes ámbitos o asignaturas para que las actividades no sean repetitivas, sino secuenciales e imbricadas con saberes anteriores y posteriores. Esto favorece al estudiante cuando la educación tiene cimientos empresariales, pues la escuela es considerada una fábrica y los alumnos el producto. Así la estandarización curricular facilita una producción en serie (visión taylorista de la educación). Además, los estándares han permitido que la humanidad avance en muchos ámbitos, evitando el caos y logrando un "ordenamiento social y mundial" (Luhmann, 1996, p. 29), ya que su aplicación garantiza la calidad del producto (el estudiante). Esta es verificada tras una evaluación estandarizada en la cual el alumno demuestra, con la aprobación de un examen, la adquisición de unos conocimientos que lo habilita para el mundo laboral.

Los estándares educativos son requisitos técnicos que establecen un conjunto de normas para implementar y gestionar el currículo de cara a alcanzar la calidad deseada (Ravitch, 1996; Casassus, 1997; Heras y Casadesús, 2006; Cueva y Rodríguez, 2010). Igualmente, Ingvarson y Kleinhenz (2006, p. 265) añaden que "los estándares son herramientas para emitir criterios sobre la práctica en un contexto de significados y valores compartidos", y que asimismo son instrumentos de medida que describirán lo que será valorado (Ingvarson y Kleinhenz, 2006; NCTM, 1991). Parafraseando a Gaulín (2001), los estándares son modelos, y además "normas de calidad de un currículo" (Ravitch; 1996) que deben ser comunes y homogéneas para resultar aplicables al conjunto de la sociedad. La National Council of teacher of mathematics (1991, p. 2) los describe como "criterios por excelencia", que conducirán a la sociedad hacia un sistema organizativo más competitivo en escenarios globalizados. En consonancia con las ideas anteriores, los estándares curriculares, medibles tras una evaluación estandarizada, permiten al Estado exigir rendición de cuentas, además de conocer lo que se espera que los profesores enseñen y los estudiantes deban aprender (Casassus, 1997).

Por tanto, al plantear los estándares curriculares se persigue homogeneizar ciertas prácticas educativas y sus procesos. La tesis expuesta por Luhmann (1996) subraya que la homogeneización consiste en proyectar un cosmos de elementos esenciales que no puede modificarse porque todo viene dado; su objetivo es ordenar la razón e intentar transformar un Estado imperfecto en otro perfecto. No obstante, aunque la homogeneización es una "construcción artificial como la geometría" (Luhmann, 1996, p. 29), los estándares intentarán responder a las exigencias mundiales; a fin de cuentas, la sociedad es la encargada de valorar su funcionalidad, ya que solo un sistema autorreferente no requiere estándares. Sin embargo, a juicio de otros (Estado, padres, estudiantes, sociedad en general), la educación requiere parámetros que ayuden a medir su calidad, para solicitar a la escuela rendición de cuentas sobre las acciones llevadas a cabo y alcanzar los objetivos deseados por la sociedad. Así, la estandarización del currículo ordena las asignaturas básicas y sus 
contenidos para ser impartidos por los profesores y directivos. De esta manera, se permite en apariencia que la humanidad avance en numerosas disciplinas; de lo contrario, todo sería caótico.

Contar con estándares supone un avance en la calidad de un servicio, como es en este caso el educativo. Sin embargo, el individuo termina como un producto elaborado de manera meticulosa que supera controles de calidad susceptibles de ser analizados (Rodríguez, 1995; Heras y Casadesús 2006; Cueva y Rodríguez, 2010). Igualmente, Ingvarson y Kleinhenz (2006) exponen que en países como Australia, los profesores y sus asociaciones ven de manera positiva el uso de estándares, al considerarlos un medio para mejorar la enseñanza y garantizar la calidad educativa, ya que ambos objetivos guardan una estrecha relación con los resultados satisfactorios de los estudiantes.

Lo anterior se refleja en la investigación de Niño y Gama (2013) en dos centros educativos de Colombia. Entre los resultados del estudio, los autores señalan que el uso de estándares permite la libre movilidad de los alumnos entre colegios y entre ciudades, y que asimismo facilita la concreción del programa de estudios y la unificación de criterios, temáticas y conceptos sobre una base conceptual homogénea.

De acuerdo con la investigación de Niño y Gama (2013), el uso de un currículo estandarizado asegura lo que el profesor va a enseñar. Igualmente, la estandarización de los contenidos describe qué debe aprender el alumno y lo bien que debería aprender, pues la función de los estándares es describir de manera clara y especifica aquellas destrezas y conocimientos que todo estudiante debe alcanzar (Ravitch, 1996; Ingvarson y Kleinhenz, 2006). Estos estándares son básicos, pues se determinan en función de la necesidad de que todos lleguen a un mínimo imprescindible (Ravitch, 1996; Casassus, 1997). Entonces, la implementación de estándares en la enseñanza ofrece ventajas, pues asegura la calidad educativa. El centro educativo adquiere un compromiso social cuyo cumplimiento es medible a través de evaluaciones (internas y externas). Además, tanto las familias como la sociedad saben qué pueden y deben aprender los estudiantes. A su vez, el Estado se asegura que el espacio comercial cuente con profesionales competentes. Por otro lado, los estándares aplicados a la educación proporcionan voz y voto a los profesores a la hora de definir y limitar su naturaleza y ámbito de trabajo. Esto significa que todas aquellas actividades que en los últimos años han invadido el terreno laboral de los profesores, tales como asumir ciertas necesidades sociales y trámites burocráticos, deberían pasar a mano de otros profesionales, de tal manera que los docentes se centren en exclusiva en todo lo relativo a su labor para aumentar la efectividad laboral y su desarrollo profesional. En otras palabras, los estándares coadyuvan a redefinir los programas de formación para los maestros y propenden a su mejora (Ingvarson y Kleinhenz. 2006).

En conclusión, el uso de estándares en el currículo educativo promueve el aprendizaje de los estudiantes, con niveles altos de rendimiento. Por tanto, su uso garantiza que todos accedan a los mismos programas con iguales expectativas y con profesorado bien preparado. No obstante, como se puede inferir, los estándares permiten que los centros educativos se comparen entre sí, en ocasiones sin una investigación profunda de su contexto de acción, para conocer si el rendimiento del estudiante se adecúa a las expectativas sociales. La escuela sigue siendo producto de una industrialización que cada vez se desarrolla con más fuerza y se encarga de preparar a la población como mano de obra competitiva en el escenario laboral. Según el clamor liberal, lo más importante es saber si los alumnos están aprendiendo tanto como pueden o deben, a fin de prepararse para una educación universitaria o técnica que cada día es más exigente debido a los acelerados cambios tecnológicos. Todo ello demanda a los ciudadanos mayores conocimientos y destrezas. Así pues, con la incorporación de estándares a la educación cobra importancia la educación instruccional y se ve menguado el aprendizaje heu- 
rístico. Como resultado, los centros educativos deben emprender un proceso de mejora continua que permita elevar el rendimiento académico de los estudiantes y encabezar la lista del ranking de centros educativos.

\section{La otra versión de los estan- darés educativos: la homoge- neización cultural}

Ciertamente el currículo estandarizado ofrece ventajas ciertamente. Sin embargo, el intento de búsqueda de la calidad educativa termina por homogeneizar los procesos de enseñanza. Estos se tornan rutinarios, mecánicos, y como contrapartida, priorizan los resultados (alto rendimiento escolar, demostrado en números) por encima de los procesos. Por ejemplo, las evaluaciones estandarizadas PISA no evalúan procesos, sino resultados obtenidos por los estudiantes en áreas del conocimiento básico (ciencias, matemáticas y lectura). Sin embargo, dejan de lado otras áreas y aspectos de la educación igual de importantes: arte, música, factores relacionados con las actitudes y valores (Santos, 2016). De este modo lo que se espera de los alumnos no es nada más que acumulen conocimiento a ser aprobados luego de evaluaciones estandarizadas.

Las diferentes investigaciones científicas sobre el tema, por ejemplo la de Lupton y Thrupp (2013) en el contexto europeo, señalan que las políticas educativas deben ser más contextualizadas para mejorar las escuelas ubicadas en zonas desfavorecidas. Esto conlleva crear nuevos estándares para centros educativos ubicados en estos contextos con el fin de hacer visibles las similitudes y diferencias entre escuelas. En la actualidad los indicadores para evaluar las escuelas son limitados, pues están centrados en medir y comparar el rendimiento escolar. Los estándares deben ser mucho más alcanzables para cada institución educativa. Además, deben ser identificadores de falencias educativas para luego trabajar en su mejora a través del intercambio de experiencias con otras instituciones ubicadas en contextos similares. Retomando la investigación de Niño y Gama (2013), realizada en países de nuestro entorno (Colombia), los estándares se derivan de políticas neoconservadoras y neoliberales propias de la globalización para controlar el saber y los sistemas educativos mundiales y nacionales. Estas ideologías, al igual que las organizaciones, por ejemplo la OCDE, ejercen influencia en los diferentes países y promueven la medición y comparación de los saberes. Por tanto, coadyuvan a la tecnificación de la enseñanza y la instrumentalización de las evaluaciones, desconociendo las diferencias contextuales y la multiculturalidad estudiantil, pues imperan más las ideas de eficiencia, eficacia y competitividad. De esta manera se fragmentan las diferentes disciplinas, sesgando el valor de la diversidad y segregando áreas del saber, ya que no son consideradas fundamentales. Sin embargo, conseguir unos mínimos imprescindibles supone medir conocimientos a través de un currículo preestablecido, pues el profesor técnico se encargará de aplicar las normas sobre los estándares. En suma, de acuerdo con la investigación de Niño y Gama (2013, p. 196), los estándares promueven una "desprofesionalización y despedagogización" de la tarea docente.

En consecuencia, esta homogeneización curricular en primera instancia beneficiaría a aquellas instituciones que tienen más similitudes y pocas diferencias entre sí. Significa que un currículo estandarizado sólo tiene sentido en escuelas ubicadas en contextos similares y con una realidad parecida. De la misma forma, Luhmann (1996) y Arriaga (2003) admiten que un sistema (en este caso la escuela como sistema abierto) no se observa desde afuera, ni basta con evaluar sus logros del mismo modo, sino desde adentro, pues cada escuela, a partir de su idiosincrasia, tiene un conocimiento de sí mismo. En efecto, una posible lectura externa que se haga acerca del rendimiento académico de los estudiantes, y por ende de la calidad educativa no es más que una aproximación a la vida de los centros, pero no describe la realidad existente en los mismos; de ahí que los procesos de mejora exigidos desde fuera pue- 
den distar mucho de lo que realmente necesita la institución educativa, pues cada escuela tiene una lectura diferente de sí misma que en muchos casos no reflejan las evaluaciones estandarizadas, por lo que evadir esa realidad es abocar a los centros a la exclusión o marginación. De esta realidad emergen dos contrastes sociales: riqueza y pobreza. En otras palabras, existirán estudiantes con más ventajas sobre otros, centros educativos con mejores niveles de aprendizaje en comparación a otros. Sin embargo, no será por falta de capacidad intelectual del alumnado, ni por la falta de preparación de sus profesionales, sino por las facilidades que ofrece el contexto social para la ejecución del currículo. En concreto, nos encontramos ante unas políticas educativas que han unificado unos criterios de enseñanza, pero que a su vez vigilan, hacen un seguimiento y una estimación de rendimientos, de modo que solo aquellas escuelas con indicadores de rendimientos elevados merecerán la pena, y así serán los estudiantes los responsables de llevar a la institución en la dirección correcta, pues confeccionan una empresa de sí mismos (Apple, 2002).

Algo parecido sostiene Popkewitz (2013) en su crítica sobre las pruebas PISA. El autor manifiesta que estas evaluaciones pretenden transformar el conocimiento que debe tener un estudiante sobre una disciplina, en conocimiento pedagógico. Es decir, los conocimientos que se miden en PISA no guardan ninguna relación con las prácticas de las disciplinas científicas. En efecto, los aprendizajes que debe adquirir un estudiante no se pueden ajustar a instrucciones técnicas, y mucho menos a un sistema de medición, ya que a estas prácticas subyacen etiquetas sobre "quién es o no es el niño, y quién debe ser" y sobre los modos de vida que se dominan y/o se deberían dominar. En consecuencia, se termina haciendo comparaciones entre estudiantes en el marco de un orden preestablecido relacionado con el modo de actuar y pensar de los alumnos, y no se valora un trabajo académico real desde la diversidad (Popkewitz, 2013, p. 19) a partir del conocimiento contextual de las escuelas. El centro educativo que refleje mejores resultados tras las evaluaciones nacionales e internacionales, será catalogado como efectivo. Sin embargo, aquellos cuyos contextos sociales les hagan más difícil llegar al cumplimiento del estándar, serán tildados como fracasados. Por tanto, la estandarización curricular pretende homogeneizar lo diverso, y las evaluaciones pretenden comparar lo incomparable, pues no podemos aislar, y aún menos obviar, la existencia de una sociedad multiculturalmente diversa ni la influencia del contexto en los centros educativos.

En concordancia con la idea anterior, Gimeno (2004) señala que la evaluación no espera a los lentos, pues los tiempos en la educación también son estandarizados. Si evaluamos a todos los estudiantes con los mismos indicadores y con tiempos limitados, se premia a los rápidos y se penaliza a los lentos. Por consiguiente, el éxito de una institución educativa no puede depender de la información que proporcionan las evaluaciones, sean estas internas o externas. Al contrario, hay que apelar a otros criterios mucho más justos y éticos, ya que en educación no todo es evaluable. Precisamente los elementos no susceptibles de evaluación, al final, son los más importantes, y esto demuestra que la estandarización impone las reglas del juego en la enseñanza colectiva, en el currículo oficial y en el funcionamiento de las instituciones (Gimeno, 2008).

Los estándares, además de homogeneizar, tienen un enfoque técnico- instrumental para medir los logros básicos y comunes que deben alcanzar los estudiantes. Esta tendencia permite a los gobiernos imponer valores (adoctrinando y controlando las mentes de los estudiantes) con tendencias ideológicas que benefician a ciertos grupos. Al imponer estos patrones, también se trivializa la enseñanza y se uniforman las aulas, debilitando la práctica docente y la transformación pedagógica. Debido a ello, el profesor se ve como un funcionario que aplica normas y disposiciones ajenas a su labor cotidiana. Cuando se aplican los estándares, lo que se persigue es igualar las escuelas desconociendo diferencias contextuales y culturales, porque se priorizan contenidos y se dejan de lado el carácter crítico de la educación y 
el fortalecimiento de las actitudes (solidaridad, el amor al prójimo, empatía y el compromiso colectivo) como parte de la formación integral del ser humano. Estos factores no son medibles en una evaluación estandarizada (Niño y Gama, 2013), pues parafraseando a Popkewitz (2013) no existe un todo universal e indiferenciado, sino particularidades que se ven manifestadas en los valores culturales de cada individuo.

Bernal (2014) acota que una manera de homogeneizar es estructurar las clases por niveles, pues esto hace que el espacio de aprendizaje sea fácil de controlar por parte de los profesores. Como años atrás argumentó claramente Gimeno (2000), con la agrupación de los estudiantes (en niveles, por edad, entre otros criterios) se pretende normalizar lo culturalmente diverso. De esta manera se aumentan y se justifican los estándares en el currículo, e incluso la forma de trabajar de los profesores, y esta situación da lugar a una carencia de variedad de los ambientes de aprendizaje y a poca innovación en los mismos. Las prescripciones curriculares sustentadas en estándares, sumadas a las políticas educativas para la implementación de los mismos, dejan claro lo perverso de la búsqueda de una homogeneización cultural en los alumnos (Molina, 2007). En definitiva "cualquier pretensión de homogeneidad apoyada en la clasificación de sujetos singulares, por tantos motivos, es un imposible" (Gimeno, 2000, p. 88), y además es rechazable por la idea de jerarquización que al final etiqueta a los no clasificables. Sin embargo, puede ser que los alumnos aprendan conocimientos de forma rápida, pero esto beneficia a los hábiles y perjudica a los más débiles. En estos últimos renacerán actitudes violentas como respuesta a una posible exclusión. No obstante, cuestiones como tolerancia, igualdad, respeto, esenciales para la formación del estudiante, no serán interiorizadas del mismo modo que los conocimientos, porque estos se viven y se practican en la relación con los otros: compañeros de clase, profesores, padres, madres, comunidad escolar en general.

En consecuencia, al homogeneizar los aprendizajes y formular estándares como instru- mentos de medición, el sistema termina segregando y clasificando a los estudiantes. A su vez se tayloriza el currículo y los tiempos de enseñanza-aprendizaje. Mientras tanto, se especializa a los profesores, como si de cualquier profesión se tratase. Dicho de otra manera, hablamos de control estatal, dejando claro en manos de quién está el poder (Morey, 1978; Apple, 2002). Así que el discurso de igualdad estará centrado en el hecho de que todos los alumnos deban aprender exactamente lo mismo, con iguales periodos de tiempo y en idéntica proporción, algo que resulta utópico y contradictorio, porque al final los procesos de evaluación internos y externos terminan comparando grupos y distribuyéndolos según los criterios que se consideren para la formación de una "cultura pública", los cuales están presentes en las disciplinas científicas y sirven como canon a la hora de construir los estándares (Pérez, 1995, p. 7). Una buena enseñanza logra ser educativa si ayuda a la transformación de nuestro conjunto de ideas, pero si nos quedamos en proporcionar conocimientos para actuar con eficiencia sobre los medios de producción, aumentarán las riquezas materiales pero sin conciencia emancipadora. La educación debe contribuir a cultivar hombres libres, con razón, y dotados de principios. De modo que la superación de estándares a manera de recta numérica puede tender al fracaso del maestro y a la frustración del estudiante.

La estandarización curricular contribuye a la homogeneización de la enseñanza pues desvirtúa los principios que deben regir la existencia de una diversidad cultural, ya que contribuye a la genética social de una cultura única. Para lograr dicha unificación se utilizan los estándares como indicadores de las dosis mínimas de información que cada individuo debe tener en su ADN social, de modo que su conciencia como sujeto es tomada y ocupada por los postulados ideológicos técnico-empresariales, lo que es propio del mundo globalizado. No cabe duda de que los caminos rectos permiten conseguir logros de manera rápida con el máximo ahorro de recursos, y los estándares dan esa seguridad si se desarrollan 
de manera uniforme; pese a ello, se reducen sus resultados, así como también la reflexión acerca de sus objetivos y logros, pues en ocasiones no se tienen en cuenta la heterogeneidad del alumnado y la complejidad del contexto social donde se aplicarán. Como se puede inferir, la educación, que representa un camino serpenteado, con curvas y desniveles que en ocasiones son muy pronunciados, es y debe ser un descubrimiento diario.

La cristianización de un currículo saneado y homogeneizado se convierte en manual para catequesis cuya finalidad es preparar a los individuos, quienes serán bautizados como aptos o no aptos para insertarse en una sociedad en la que ya están inmersos desde su nacimiento. Partiendo de esto, la escuela también es utilizada para extender preceptos morales, y no verdadero conocimiento, pues el individuo, como todo enfermo, recibe un tratamiento que lo hará más controlable y menos libre. Bajo estos condicionamientos, la educación pierde su valor de fuerza social transformadora (Castel y Foucault, 1981). En otras palabras, lo que se esconde detrás de una estandarización curricular son números que se "enmarcan en un conjunto de prácticas que generan una tesis cultural sobre quién es el niño y quién debe ser" (Popkewitz, 2013, p. 59). A causa de ello la escuela, con la ayuda del currículo estandarizado, fabrica al estudiante sobre unos cánones sociales preestablecidos por aquellos que creen ser poseedores de la cultura única.

De lo anterior se puede inferir que la escuela es una "prisión sin barrotes" de "educación vigilada" en la que los centros no son más que islotes controlados desde el exterior (Grignon, 1981, p. 65). Ella dispensa una enseñanza teórica y menos acorde con el contexto donde el estudiante desarrolla su día a día, traduciéndose en libresca y superficial, en un almacén de fechas, nombres y eventos registrados y grabados en la memoria cuyo aprendizaje se comprueba por medio de un examen (Giroux y McLaren 1998; Santos, 1994). Sin embargo, para entender y reconocer las necesidades e intereses reales de la ciudadanía con respecto a sus saberes particulares, los estándares como instrumento para igualar conocimientos resultan contradictorios y dejan rendijas hacia un aprendizaje superficial y vacío, pues al final se centran en comparaciones que dejan emerger más las diferencias que las similitudes. En consecuencia, las personas se destacarán por el volumen de información que almacenan y guardan en su memoria más que por su ingenio y por su capacidad de innovar y de resolver problemas cotidianos. Esto hará personas competitivas entre sí, pero carentes de generosidad, empatía y faltas de sensibilidad ante el dolor y el sufrimiento ajeno. Igualmente, manifestarán poco amor y cuidado hacia la naturaleza, y además olvidarán que el bienestar de la mayoría se encuentra por encima del bienestar individual (propio de la cultura capitalista).

Otra desventaja de la homogeneización son las escuelas con apariencia de fábrica de orden taylorista de carácter científico, porque, en palabras de Blázquez (1999, p. 79), "vivimos en una sociedad en la que, con demasiada frecuencia, se sacralizan valores como la eficiencia, la fiabilidad, la versatilidad o la rapidez" al servicio de los poderes neoliberales que hacen del verdadero trabajo docente y directivo algo administrativo y burocrático. Ello da a lugar a "responsabilidades de trabajo más pesadas y requerimientos siempre crecientes de informes, planificación de reuniones sin término $y$, en muchos casos, una creciente escasez de recursos, tanto emocionales como físicos", cosas inútiles que no responden a las necesidades reales de una sociedad diversa y multicultural (Apple, 2002, p. 234). Al respecto, Santos (1999, p. 27) subraya que "la escuela no es una empresa, ni en la naturaleza de su tarea, ni en los fines, ni en las exigencias ideológicas, políticas y morales", porque el sentido empresarial de la escuela evoca una enseñanza rutinaria y mecánica. Por tanto los directores y los profesores son, en parte, instrumentos utilizados para poner en funcionamiento toda esta maquinaria científica; los estudiantes se convierten en productos tecnificados susceptibles de ser patentados. Esta tesis es valorada por Apple (1986, p. 148), al señalar 
que "la escuela es la planta procesadora y el hombre educado es el producto". Asimismo, RattrayWood y Parrott (1989) puntualizan que los padres también aprueban estos programas educativos de ideología técnico-empresarial, pues esto es lo que les permite a sus hijos incursionar en el mercado laboral. En conclusión:

[...] pretender una homogenización sencillamente no va a funcionar. Necesitamos preguntarnos constantemente qué significan las reformas para las escuelas en su conjunto $y$ para cada uno de sus participantes, incluidos profesores, estudiantes, administradores, miembros de la comunidad, activistas locales y demás. Se gasta más tiempo y energía en mantener y mejorar una imagen pública de una buena escuela y menos tiempo y energía en materia pedagógica y curricular (Apple, 2002, p. 232).

Igualmente, no cabe duda de que todos los individuos deben tener las mismas oportunidades para el acceso a la información y la búsqueda del conocimiento. Sin embargo, no hay que obviar la existencia de formas culturales diversas. Por ello, surge la necesidad de políticas educativas flexibles que orienten y exijan rendición de cuentas a los actores educativos, pero dejando los mínimos o máximos a alcanzar en manos de docentes y directores, que son los más cercanos al niño y a su entorno.

\section{Consideraciones finales}

La aplicación de un currículo estandarizado obliga a que su uso se lleve a cabo de manera uniforme, con procesos de enseñanza en recta numérica. Por tanto, se cae en una construcción cultural homogénea, exigiendo al ser humano adaptarse a los estándares, y no estos a él. La estandarización curricular pretende formar hombres y mujeres modélicos con ideología técnico-empresarial.

El pretender homogeneizar el currículo exige de los centros educativos una organización de visión educativa empresarial de enseñanza taylorista. Sin embargo, la escuela no se puede comparar con este tipo de estructura, pues la diana de la educación es la formación del hombre de manera integral (conocimiento, práctica y valores) para, con ello, procurar una sociedad mejor y el desarrollo de la misma.

Por otro lado, el implementar algún tipo de estandarización curricular supone estudiar, antes, el contexto inmediato en la cual se lleva a cabo el quehacer educativo, para identificar los factores externos que subyacen al mismo. Estos tropismos, en algunas ocasiones, suponen un punto de inflexión para la implementación de cualquier sistema de rendición de cuentas en los centros educativos, que tenga como imperativo solo medir resultados de aprendizajes con instrumentos homogéneos para realidades que en esencia son diversas. Esto quiere decir que si bien es de justicia que el Estado y el conjunto de la sociedad conozcan qué se enseña y cómo se enseña en la escuela, esto no puede ser a costa de obviar las grandes discrepancias que existen entre un contexto y otro, pues precisamente esas diferencias son las que desmantelan cualquier idea de estandarización curricular. Esta estandarización no siempre resulta ser un instrumento justo para medir resultados de aprendizaje y equiparar oportunidades cuando en la realidad las instituciones difieren en figura y fondo unas de otras porque el componente poblacional de la escuela es culturalmente diverso. Además, una parte de las instituciones educativas están ancladas en contextos complejos agravados por su problemática social.

No obstante, si la norma es que todo conocimiento es y debe ser medible, entonces los instrumentos y técnicas que se creen para este fin necesitan de una rigurosa contextualización en la realidad de cada centro educativo, teniendo en cuenta que algunos factores del contexto externo sí influyen en los procesos internos de los centros educativos. En suma, una educación con visión capitalista que hace que el individuo se encuentre inmerso en un mundo de competitividad (entre centros educativos y entre estudiantes) y constantemente selectivo (centros educativos con baja calificación son considerados de difícil desempeño), sitúa a los centros educativos en el 
escaparate de las industrias, que respeta y supera cada estándar como si de un control de calidad se tratase, alejándonos del imperativo categórico que persigue la educación: formar hombres y mujeres innovadores, que no se limiten a repetir lo que otros profesan como verdad universal.

Es preciso cuestionarnos a quién debe realmente rendir cuentas el sistema educativo, y el simple hecho de hacerlo supone revisar y redefinir el currículo, la pedagogía, la planificación, los métodos y los procesos de evaluación adecuados a los diferentes contextos que, en apariencia, simulan ser uniformes, pero que en el fondo guardan grandes diferencias entre sí. Esto también significa alejarnos de lo libresco que aún persiste en la enseñanza y de su selectividad en cuanto a sus exigencias estandarizadas, pues tantos paquetes curriculares y evaluadores homogeneizadores de los sistemas educativos, terminan siendo un manual de catequesis para un proceso de evangelización y, por tanto, contraproducentes y culturalmente destructivos para los individuos y para la sociedad.

\section{Referencias bibliográficas}

Apple, M. (1986). Ideología y currículo. Madrid: Akal Ediciones.

Apple, M. (2002). Mercados, estándares y desigualdad. ¿Pueden las pedagogías críticas para las políticas derechistas? Revista de educación, Número extraordinario, 223- 248.

Arriaga, E. (2003). La Teoría de Niklas Luhmann. Convergencia. Revista de Ciencias Sociales, 10(32), 277-311. Recuperado de https:// goo.gl/Pn3MBr

Bernal, J. (2014). El centro escolar: espacios y procesos. Organización de los centros educativos. LOMCE y políticas Neoliberales. Zaragoza: Mira Editores.

Blázquez, F. (1999). Los proyectos institucionales, un espacio para la educación intercultural, En Organización y Dirección de Instituciones Educativas en Contextos Interculturales (pp. 74-94). Granada: Universidad de Granada.

Casassus, J. (1997). Estándares en educación: Conceptos y fundamentales. Santiago:
OREALC/UNESCO. Recuperado de https://goo.gl/161nDu

Castel, R., \& Foucault, M. (1981). Espacios de Poder. Madrid: La Piqueta.

Cueva, S., \& Rodríguez, G. (2010). OER, Estándares y Tendencias. Revista de Universidad y Sociedad del Conocimiento. 7(1), 1-8. Recuperado de https://goo.gl/27Xf1m

Freire, P. (1990). La naturaleza política de la educación: cultura, poder y liberación. Barcelona: Paidós.

Gaulín, C. (2001). Tendencias actuales de la resolución de problemas. Conferencia pronunciada en la EusKalduna, Bilbao. Sigma, 19, 51-63. Recuperado de https://goo.gl/dwjwbj

Gimeno, J. (2000). La educación obligatoria: su sentido educativo y social. Madrid: Ediciones Morata.

Gimeno, J. (2004). Quién fracasa cuando hay fracaso escolar? Recuperado de https://goo.gl/YGJDNU

Gimeno, J. (2008). El valor del tiempo en la educación. Madrid: Ediciones Morata.

Giroux, H., \& McLaren, P. (1998). Educación de maestros y la política del compromiso: el caso pro-escolarización democrática. En Sociedad, cultura y educación (pp. 87- 136). Buenos Aires: Miño y Dávila.

Grignon, C. (1981). La enseñanza agrícola y la dominación simbólica del campesinado. En Espacios de poder (pp. 53-78). Madrid: La Piqueta.

Hargreaves, A., \& Fullan, M. (2014). Capital profesional. Madrid: Morata.

Heras, I., \& Casadesús, M. (2006). Los estándares internacionales de sistemas de gestión. Pasado, presente y futuro. Boletín económico de ICE, 2876, 45-60. Recuperado de https:// goo.gl/1vLccd

Ingvarson, L., \& Kleinhenz, E. (2006). Estándares profesionales de práctica y su importancia para la enseñanza. Revista de Educación, 340, 265-295. Recuperado de https://goo.gl/U5MYkp

Luhmann, N. (1996). Teoría de la sociedad y pedagogía. Barcelona: Paidós.

Lupton, R., \& Thrupp, M. (2013). Headteachers' readings of and responses to disadvantaged contexts: evidence from English primary schools. British Educational Research Journal, 39(4), 769-788. https://doi.org/:10.1080/01411926.2012.683771 
Molina, S. (2007). La escolarización obligatoria en el siglo XXI. Madrid: La Muralla.

Morey, M. (Ed.) (1978). Sexo, poder, verdad. Conversaciones con Michael Foucault. Barcelona: Cuadernos Materiales.

Muñoz, C., Córdoba, J., \& Villareal, J. (2014). Estándares Internacionales de Educación (IES) en contabilidad y aseguramiento: Nuevos retos de la profesión contable. Tendencias, 15(2), 118-135. https://doi.org/10.22267/rtend.141502.45

National Council of Teachers of Mathematics (NCTM). (1991). Estándares curriculares y de evaluación para la educación matemática. Sevilla: SAEM Thales.

Niño, L., \& Gama, A. (2013). Los estándares en el currículo y la evaluación: ¿relaciones de medición, control y homogenización o, posibilidad de formación, diversidad y evaluación crítica?. Revista Electrónica Interuniversitaria de Formación del Profesorado, 16(3), 185-198. Recuperado de https://goo.gl/PF6vVT

Pérez (1995). La escuela, encrucijada de culturas. Revista Investigación en la Escuela, 26, 7-24. Recuperado de https://goo.gl/qCkDkn

Popkewitz, T. (2013). Pisa: Números, estandarización de la conducta y la alquimia de las materias escolares. Profesorado: Revista de currículum y formación del profesorado, 2(17), 47-64. Recuperado de https://goo.gl/T7nD9m

Quijano, O. (2005). Sociedad y gestión del conocimiento: Los eufemismos del capitalismo cognitivo. Una lectura del mercado del conocimiento para nuestro mundo inmediato. En Encuentro Nacional de Investigación en Educación, (pp. 205-228). Recuperado de https://goo.gl/46rX9i

Rattray-Wood, L., \& Parrott, J. (1989). Crisis de la sociedad y Administración Educativa. En Práctica crítica de la administración educativa (pp. 27-73). Valencia: Universitat de Valencia.

Ravitch, D. (1996). Estándares Nacionales en Educación. Santiago: PREAL. Recuperado de https://goo.gl/qbTh1Q

Rodríguez, T. (1995). Poder y saber (La Micropolítica Foucaultiana y la Práctica Escolar). Teoría de la Educación, 7, 163-181. Recuperado de https://goo.gl/cvTqfv
Santos, M. (1994). Entre bastidores. El lado oculto de la organización escolar. Málaga: Ediciones Aljibe.

Santos, M. (1999). Organizaciones que aprenden. En Organización y dirección de instituciones educativas en contextos interculturales (pp. 21-37). Granada: GEU.

Santos, M. (2016, diciembre 10). PISA o la cesta de piedras. En La opinión de Málaga. Recuperado de https://goo.gl/ssJc9h 